\title{
Knockdown of PREX2a inhibits the malignant phenotype of osteosarcoma cells
}

\author{
ZIXUN DAI, JIE XIE, PENGFEI LEI and YIHE HU \\ Department of Orthopedics, Xiangya Hospital of Central South University, Changsha, Hunan 410008, P.R. China
}

Received February 17, 2015; Accepted December 11, 2015

DOI: $10.3892 / \mathrm{mmr} .2015 .4728$

\begin{abstract}
Phosphatidylinositol-3,4,5-trisphosphate-dependent Rac exchange factor $2 \mathrm{a}$ (PREX2a), which is a regulator of the small guanosine triphosphatase Rac, has recently been reported to have an oncogenic role via the suppression of phosphatase and tensin homolog deleted on chromosome ten (PTEN) activity, and the subsequent activation of phosphoinositide 3-kinase (PI3K) signaling. However, the detailed role of PREX2a in osteosarcoma (OS) remains unclear. Reverse transcription quantitative polymerase chain reaction and western blotting was used to detect mRNA and protein levels. MTT assay was performed to examine cell proliferation and a Transwell assay and wound healing assay were conducted to examine cell invasion and migration. The present study demonstrated that PREX2a was markedly upregulated in OS cell lines as compared with in normal osteoblast hFOB1.19 cells. In addition, knockdown of PREX2a expression significantly inhibited OS cell proliferation, whereas overexpression of PREX2a markedly promoted OS cell proliferation. Inhibition of PREX2a also markedly suppressed the invasion and migration of OS cells, at least partly by suppressing the protein expression of matrix metalloproteinase (MMP) 2 and MMP9. Conversely, upregulation of PREX2a enhanced OS cell invasion and migration. In addition, PI3K signaling activity was significantly reduced following knockdown of PREX2a, and this was accompanied by an upregulation of PTEN activity. The results of the present study suggested that PREX2a may act as an oncogene in OS via the inhibition of PTEN activity and activation of PI3K signaling.
\end{abstract}

Correspondence to: Professor Yihe Hu, Department of Orthopedics, Xiangya Hospital of Central South University, 87 Xiangya Road, Changsha, Hunan 410008, P.R. China

E-mail: csuhuyihe@163.com

Key words: phosphatidylinositol-3,4,5-trisphosphate-dependent Rac exchange factor 2a, osteosarcoma, phosphoinositide 3-kinase, phosphatase and tensin homolog deleted on chromosome ten

\section{Introduction}

Osteosarcoma (OS) is the most common type of malignant bone tumor, with a morbidity of $\sim 5$ cases per million worldwide (1). Due to its resistance to radiotherapy, chemotherapy and adjuvant therapies, the median survival rate of OS has not markedly improved over the past few decades $(1,2)$. Previous studies have demonstrated that aberrant upregulation of oncogenes has a key role in the development and progression of OS $(3,4)$. Therefore, identification of novel oncogenes may have potential as therapeutic targets for OS.

It has previously been reported that aberrant activation of phosphoinositide 3-kinase (PI3K) signaling is tightly associated with numerous types of human cancer $(5,6)$. Whole-exome, whole-genome and RNA sequencing have highlighted the PI3K pathway as a common vulnerability in OS, and inhibition of PI3K signaling attenuates the malignant progression of OS (7-9). Phosphatase and tensin homolog deleted on chromosome ten (PTEN) is able to inhibit PI3K signaling activity, and is thus considered an important tumor suppressors (10). Phosphatidylinositol 3,4,5-trisphosphate-dependent Rac exchange factor 2a (PREX2a), which is a regulator of the small guanosine triphosphatase Rac, has recently been reported to act as an inhibitor of PTEN activity via directly binding to PTEN through its guanine nucleotide exchange factor (GEF) domains $(11,12)$. PREX2a is able to inhibit the lipid phosphatase activity of PTEN, leading to the accumulation of phosphatidylinositol $(3,4,5)$-trisphosphate and phosphorylation of Akt/mammalian target of rapamycin (13). Previous studies have suggested that PREX2a may have an oncogenic role in numerous types of human cancer, including gastric cancer, neuroblastoma and melanoma (14-16). However, the exact role of PREX2a in OS has yet to be elucidated.

The present study aimed to investigate the role of PREX2a in the regulation of the proliferation, migration and invasion of OS cells. In addition, the underlying molecular mechanisms were examined.

\section{Materials and methods}

Reagents. Fetal bovine serum (FBS), TRIzol ${ }^{\circledR}$ reagent, Lipofectamine ${ }^{\circledR}$ 2000, 3-(4,5-dimethylthiazol-2-yl)-2,5-diphenyltetrazolium bromide (MTT) and RevertAid First Strand cDNA Synthesis kit were purchased from Thermo Fisher Scientific, Inc. (Waltham, MA, USA). SYBR Green 
quantitative polymerase chain reaction (qPCR) assay kit was purchased from TOYOBO (Shanghai) Co., Ltd. (Shanghai, China). Mouse polyclonal anti-PREX2a (1:100 dilution; cat. no. ab169027), rabbit monoclonal anti-phosphorylated (p)-PTEN (1:200; cat. no. ab109454), mouse monoclonal anti-PTEN (1:100 dilution; cat. no. ab79156), rabbit monoclonal anti-p-PI3K (1:200 dilution; cat. no. ab182651), mouse monoclonal anti-PI3K (1:200 dilution; cat. no. ab86714), mouse monoclonal anti-glyceraldehyde 3-phosphate dehydrogenase (GAPDH; 1:100 dilution; cat. no. ab8245), mouse monoclonal anti-MMP-2 antibody (1:200 dilution; cat. no. ab86607) and mouse monoclonal anti-MMP-9 (1:100 dilution; cat. no. ab58803 antibodies, and rabbit anti-mouse IgG (1:20,000 dilution; cat. no. ab6728) and goat anti-rabbit IgG (1:10,000 dilution; cat. no. ab6721) secondary antibodies were purchased from Abcam (Cambridge, MA, USA). Enhanced chemiluminescence (ECL) kit was purchased from Pierce Protein Biology; Thermo Fisher Scientific, Inc. (Rockford, IL, USA). Cell Invasion Assay kit was purchased from Merck Millipore (Darmstadt, Germany).

Cell culture. The MG63, Saos-2 and U2OS human OS cell lines, and the hFOB1.19 human osteoblast cell line were obtained from the Cell Bank of Central South University (Changsha, China). The cells were cultured in RPMI-1640 (Thermo Fisher Scientific, Inc.) medium supplemented with $10 \% \mathrm{FBS}$ at $37^{\circ} \mathrm{C}$ in a humidified incubator containing $5 \%$ $\mathrm{CO}_{2}$.

Transfection. Lipofectamine ${ }^{\circledR} 2000$ was used to transfect the cells, according to the manufacturer's protocol. Briefly, cells were cultured to $70 \%$ confluence, and resuspended in serum-free medium. Small interfering (si)RNAs (PREX2a specific siRNA and negative control siRNA), plasmids (pcDNA3.1-PREX2a plasmid and pcDNA3.1 blank vector; Auragene Biosciences, Changsha, China) and Lipofectamine ${ }^{\circledR} 2000$ were diluted with serum-free medium. The diluted Lipofectamine ${ }^{\circledR} 2000$ was added to the diluted siRNA or plasmid, and the mixture was incubated for $20 \mathrm{~min}$ at room temperature. Subsequently, the mixture was added to the cell suspension. Following a $6 \mathrm{~h}$ incubation at $37^{\circ} \mathrm{C}$ and $5 \%$ $\mathrm{CO}_{2}$, the medium was replaced with normal serum-containing medium.

Reverse transcription (RT)-qPCR analysis. Total RNA was extracted from the cells using TRIzol ${ }^{\circledR}$ reagent, according to the manufacturer's protocol. Total RNA was reverse transcribed into cDNA using the RevertAid First Strand cDNA Synthesis kit, according to the manufacturer's protocol. mRNA expression levels were examined using the SYBR Green qPCR assay kit, in accordance with the manufacturer's protocol. The sequences of the specific primer (Sangon Biotech Co., Ltd., Shanghai, China) pairs used were as follows: PREX2a, sense 5'-TGGGAGGGGTCCAACATCA-3', anti-sense 5'-TCTTCA ACCGTCTGTGTTTTCTT-3'; GAPDH, sense 5'-CTCCTC CTGTTCGACAGTCAGC-3' and anti-sense: 5'-CCCAAT ACGACCAAATCCGTT-3'. An ABI 7500 thermal cycler was used (Applied Biosystems, Foster City, CA, USA). The reaction conditions were as follows: $95^{\circ} \mathrm{C}$ for $3 \mathrm{~min}$ and 40 cycles of denaturation at $95^{\circ} \mathrm{C}$ for $15 \mathrm{sec}$ and annealing/elongation at $60^{\circ} \mathrm{C}$ for $30 \mathrm{sec}$. GAPDH was used as an internal control. Independent experiments were repeated three times. The relative mRNA expression levels were analyzed using the $2^{-\Delta \Delta \mathrm{Cq}}$ method (17).

Western blotting. Cells were solubilized in cold radioimmunoprecipitation lysis buffer (Beyotime Institute of Biotechnology, Shanghai, China). Protein concentration was determined using a BCA Protein Assay kit (Beyotime Institute of Biotechnology). Subsequently, protein (20 $\mu \mathrm{g}$ per lane) was separated by $10 \%$ sodium dodecyl sulfate-polyacrylamide gel electrophoresis, and was transferred from the gel to nitrocellulose membranes (Thermo Fisher Scientific, Inc.). The membranes were blocked in 5\% nonfat dried milk in phosphate-buffered saline (PBS) containing $0.1 \%$ Tween for $3 \mathrm{~h}$ and were then incubated overnight at $4^{\circ} \mathrm{C}$ with mouse anti-PREX2a monoclonal antibody (1:100), mouse anti-matrix metalloproteinase (MMP)2 monoclonal antibody (1:100), mouse anti-MMP9 monoclonal antibody (1:100), mouse anti-p-PI3K monoclonal antibody (1:100), mouse anti-PI3K monoclonal antibody (1:100), mouse anti-p-PTEN monoclonal antibody (1:100), mouse anti-PTEN monoclonal antibody (1:100) and mouse anti-GAPDH monoclonal antibody (1:400). Following washing twice with Dulbecco's phosphate-buffered saline (Thermo Fisher Scientific, Inc.; $5 \mathrm{~min} /$ wash), the membranes were incubated with rabbit anti-mouse secondary antibody $(1: 5,000)$ for $40 \mathrm{~min}$ at room temperature. Subsequently, immune complexes were detected using an ECL kit. The membranes were scanned in grayscale by Image-Pro Plus software 6.0 (Media Cybernetics, Inc., Rockville, MD, USA), in order to determine the relative protein expression levels. The relative protein expression levels are presented as the density ratio vs. GAPDH.

Cell proliferation assay. The MTT assay was used to measure cell proliferation. Cells $\left(1 \times 10^{5}\right.$ cells/well $)$ in each group were cultured in a 96-well plate, each well was filled with $100 \mu \mathrm{l}$ fresh serum-free medium and $0.5 \mathrm{~g} / \mathrm{l} \mathrm{MTT}$. The plate was incubated at $37^{\circ} \mathrm{C}$ for $0,24,48$ and $72 \mathrm{~h}$, after which the medium was removed by aspiration and $50 \mu \mathrm{l}$ dimethyl sulfoxide was added to each well. Following a further $10 \mathrm{~min}$ incubation at $37^{\circ} \mathrm{C}$, the absorbance of each sample was measured at a wavelength of $492 \mathrm{~nm}$ using a plate reader (CX22; Olympus, Tokyo, Japan).

Cell invasion assay. Cells $\left(1 \times 10^{6}\right.$ cells/well) were starved in serum-free medium for $24 \mathrm{~h}$ and were subsequently resuspended in serum-free medium. The Transwell was coated in Matrigel. Cells were added to the upper chamber of a Transwell system, whereas the lower chamber was filled with medium supplemented with $10 \%$ FBS. Following a $24 \mathrm{~h}$ incubation, the cells attached to the bottom were stained with crystal violet for $20 \mathrm{~min}$, and were washed and air-dried. Invasive cells were observed under a microscope.

Wound scratch assay. Wound scratch assay was performed in order to determine the cell migratory capacity of each group. Cells were cultured to full confluence and a wound of $\sim 1 \mathrm{~mm}$ width was created in the cell layer using a plastic scriber. Subsequently, the cells were washed with PBS and 

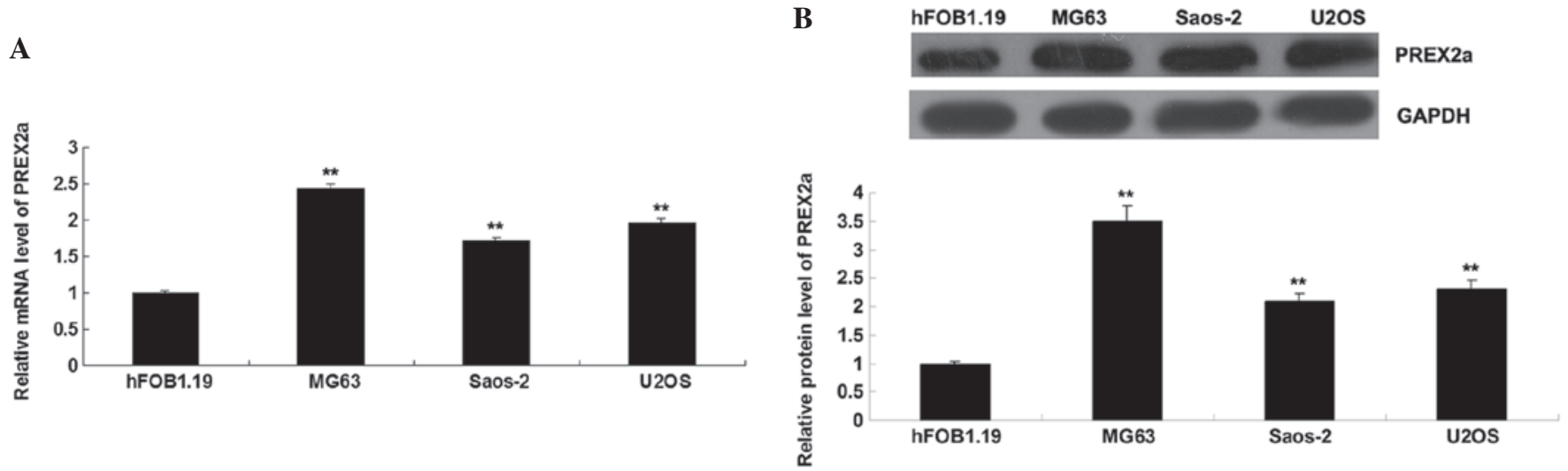

Figure 1. (A) mRNA and (B) protein expression levels of PREX2a in osteosarcoma cell lines, MG63, Saos-2, and U2OS, as determined by reverse transcription-quantitative polymerase chain reaction and western blotting, respectively. The normal human osteoblast cell line hFOB1.19 was used as a control. Data are presented as the mean \pm standard deviation. ${ }^{* * *} \mathrm{P}<0.01$ vs. the hFOB1.19 cells. PREX2a, phosphatidylinositol-3,4,5-trisphosphate-dependent Rac exchange factor 2a; GAPDH, glyceraldehyde 3-phosphate dehydrogenase.

A
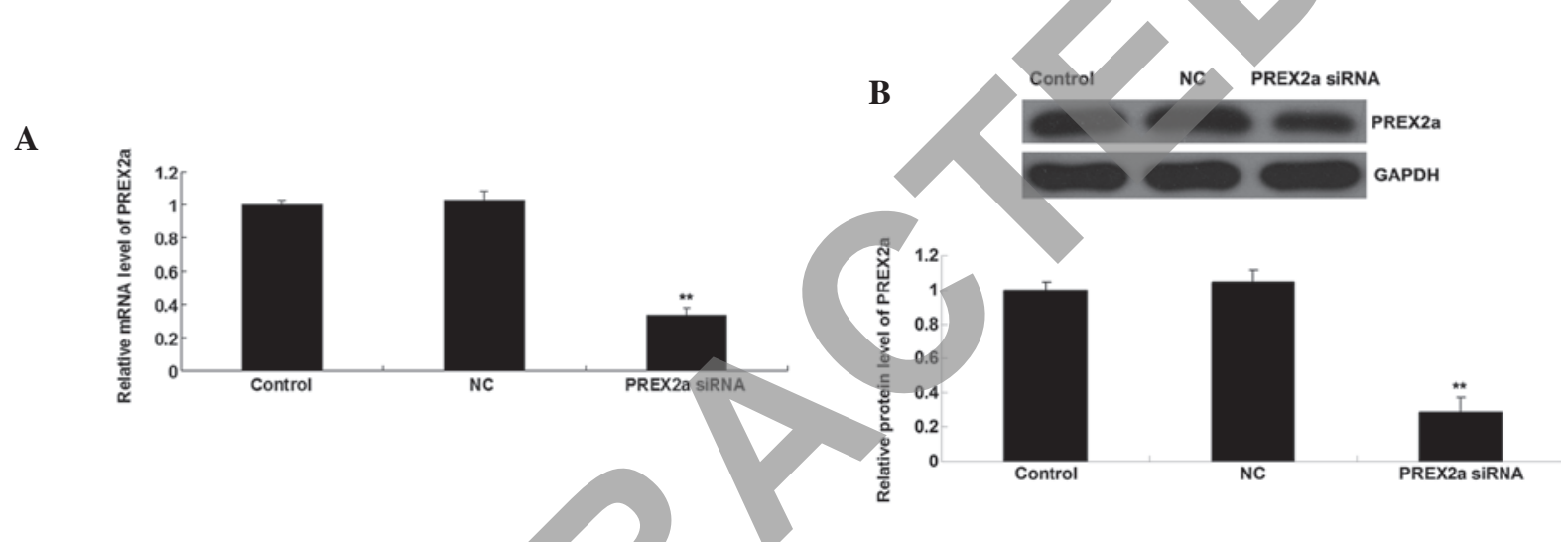

C

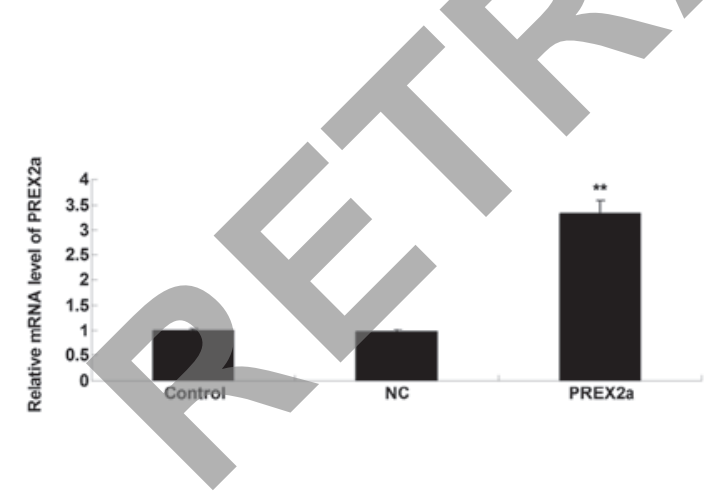

D

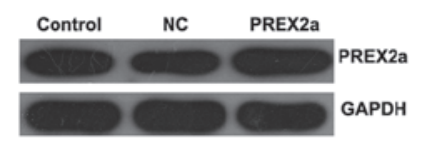

Figure 2. (A) mRNA and (B) protein expression levels of PREX2a in MG63 cells transfected with PREX2a siRNA or non-specific siRNA (NC) as detected by RT-qPCR and western blotting, respectively. (C) mRNA and (D) protein expression levels of PREX2a in MG63 cells transfected with pcDNA3.1-PREX2a plasmid or blank pcDNA3.1 vector (NC), as detected by RT-qPCR and western blotting, respectively. Control group: Untransfected cells. Data are presented as the mean \pm standard deviation. ${ }^{* *} \mathrm{P}<0.01$ vs. the control cells. PREX2a, phosphatidylinositol-3,4,5-trisphosphate-dependent Rac exchange factor 2a; GAPDH, glyceraldehyde 3-phosphate dehydrogenase; siRNA, small interfering RNA; NC, negative control; RT-qPCR, reverse transcription-quantitative polymerase chain reaction.

were incubated for $48 \mathrm{~h}$ at $37^{\circ} \mathrm{C}$ and $5 \% \mathrm{CO}_{2}$. The cells in each group were fixed with $90 \%$ ethanol and observed under a microscope.

Statistical analysis. Data are presented as the mean \pm standard deviation of three independent experiments. The results were analyzed using SPSS 17.0 statistical software (SPSS, Inc., Chicago, IL, USA). Differences between the groups were determined using one-way analysis of variance. $\mathrm{P}<0.05$ was considered to indicate a statistically significant difference.

\section{Results}

PREX2a is upregulated in OS cells. To determine the role of PREX2a in OS, the expression levels of PREX2a were detected in OS cell lines: MG63, Saos-2 and U2OS. The normal human osteoblast cell line hFOB1.19 was used as a control. As shown in Fig. 1A and B, the mRNA and protein expression levels of PREX2a were significantly increased in the OS cells, as compared with in the hFOB1.19 cells. Since MG63 cells exhibited the most significant upregulation in 
A

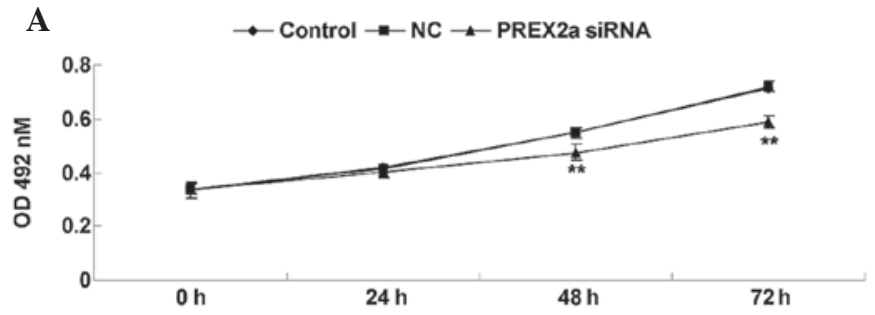

B

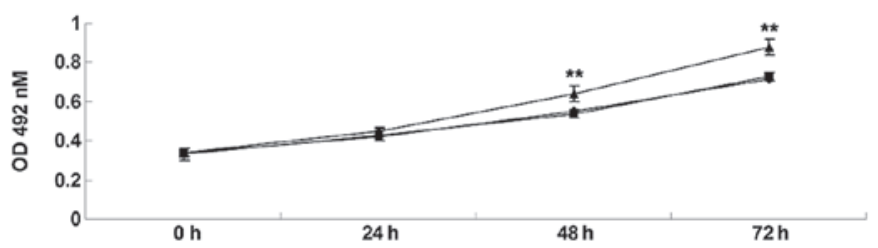

Figure 3. Cell proliferation assay was performed in (A) MG63 cells transfected with PREX2a siRNA or non-specific siRNA (NC); and (B) in MG63 cells transfected with pcDNA3.1-PREX2a plasmid or blank pcDNA3.1 vector (NC). Control group: Untransfected cells. Data are presented as the mean \pm standard deviation. ${ }^{* *} \mathrm{P}<0.01$ vs. the control cells. PREX2a, phosphatidylinositol-3,4,5-trisphosphate-dependent Rac exchange factor 2a; NC, negative control; siRNA, small interfering RNA; OD, optical density.

A
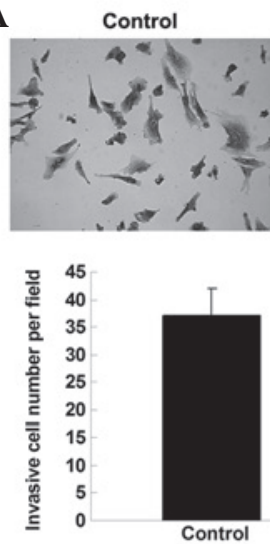

C
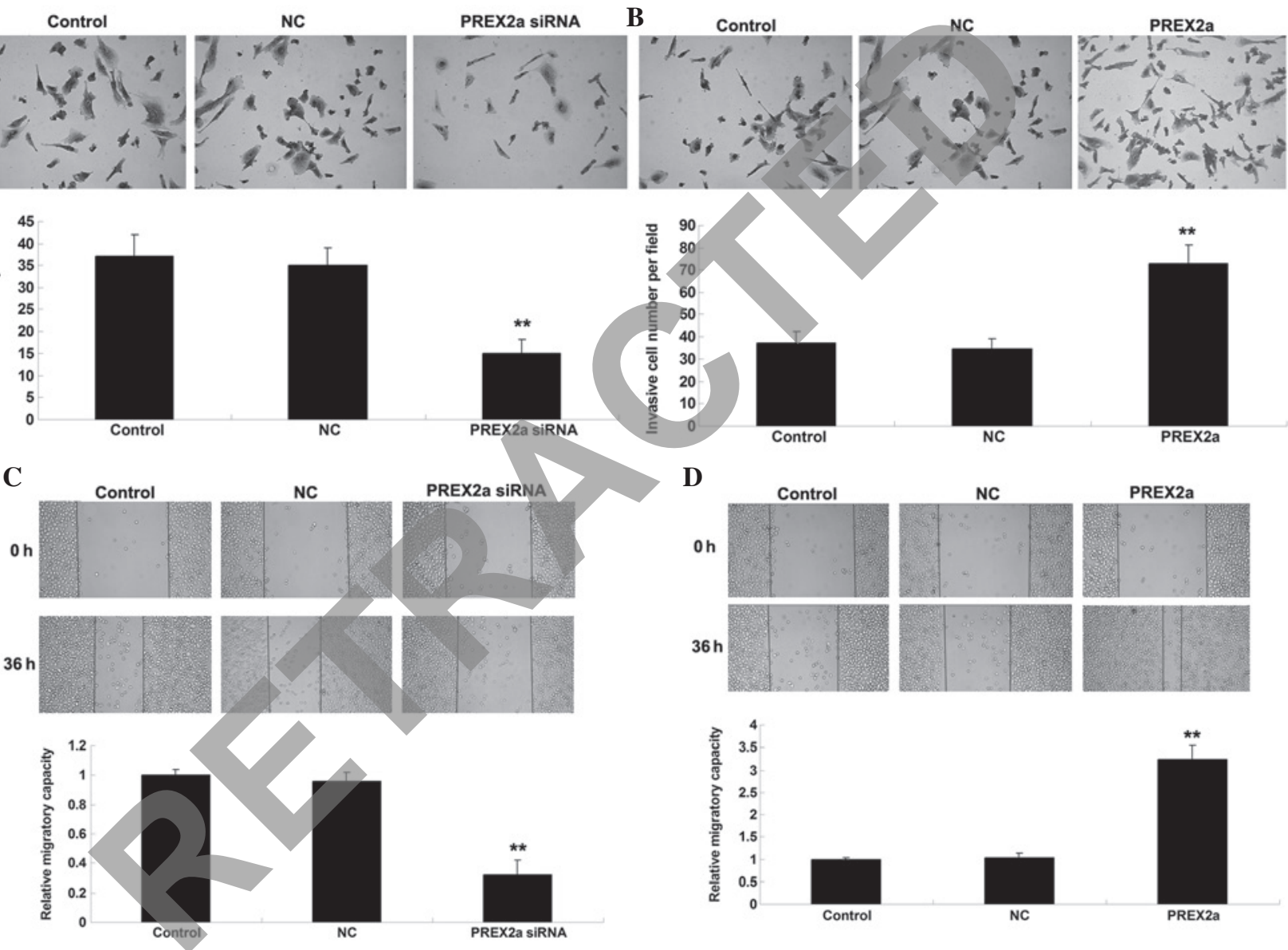

D
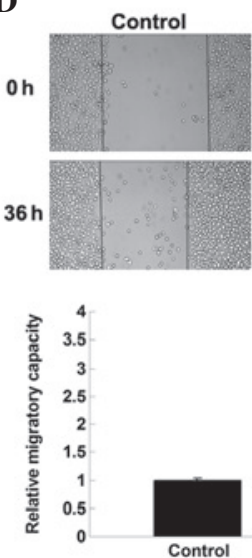

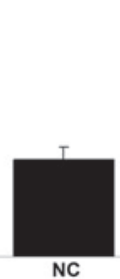

NC
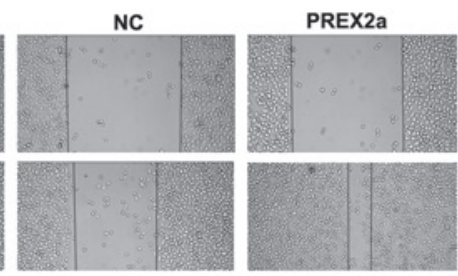

$\stackrel{2 *}{*}$

PREX2a

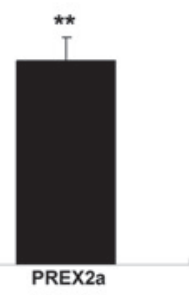

Figure 4. Cell invasion assay was performed (A) in MG63 cells transfected with PREX2a siRNA or non-specific siRNA (NC); and (B) in MG63 cells transfected with pcDNA3.1-PREX2a plasmid or blank pcDNA3.1 vector (NC; magnification, x200). Cell migration assay was performed (C) in MG63 cells transfected with PREX2a siRNA or non-specific siRNA (NC); and (D) in MG63 cells transfected with pcDNA3.1-PREX2a plasmid or blank pcDNA3.1 vector (NC). Control group: Untransfected cells. Data are presented as the mean \pm standard deviation. ${ }^{* *} \mathrm{P}<0.01$ vs. the control cells. PREX2a, phosphatidylinositol-3,4,5-trisphosphate-dependent Rac exchange factor 2a; NC, negative control; siRNA, small interfering RNA.

PREX2a expression, this cell line was used in all subsequent experiments. The MG63 OS cells were transfected with PREX2a-specific siRNA or pcDNA3.1-PREX2a plasmid. Non-specific siRNA and blank pcDNA3.1 vector were used as negative controls. Post-transfection, the protein and mRNA expression levels of PREX2a were detected in each group. The expression levels of PREX2a were significantly reduced post-transfection with PREX2a siRNA (Fig. 2A and $\mathrm{B})$; however, the expression levels were markedly increased post-transfection with the pcDNA3.1-PREX2a plasmid (Fig. 2C and D). Transfection with non-specific siRNA or blank pcDNA3.1 vector did not affect the expression levels of PREX2a. These data indicate that the transfection was successful.

PREX2a enhances OS cell proliferation. The present study determined the proliferative capacity of OS cells in each group. As shown in Fig. 3A and B, knockdown of PREX2a significantly inhibited OS cell proliferation. Conversely, overexpression of PREX2a notably promoted OS cell proliferation. These results suggest that PREX2a may promote the proliferation of MG63 OS cells. 
A
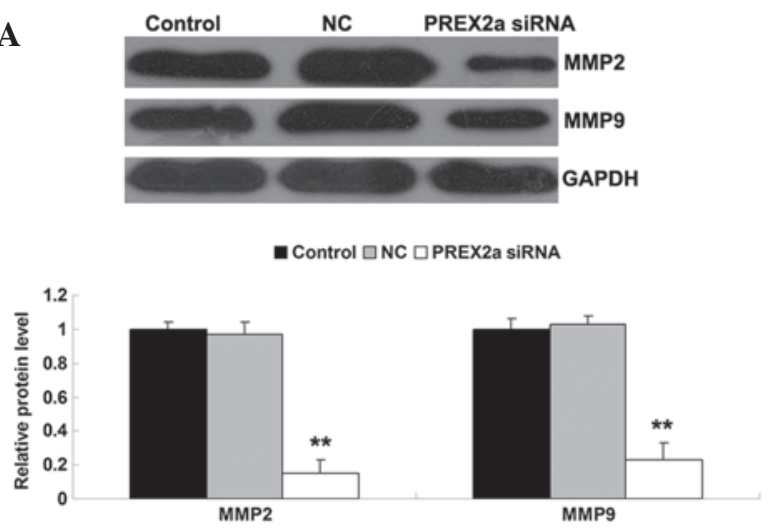

B
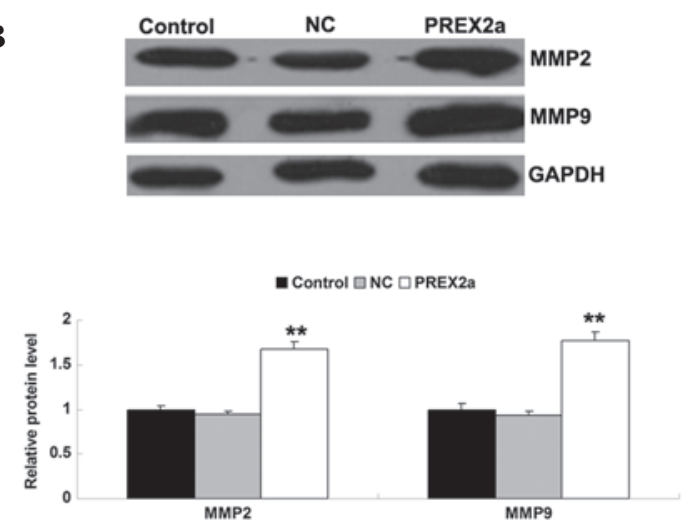

Figure 5. Western blot analysis was performed to determine the protein expression levels of MMP2 and MMP9 (A) in MG63 cells transfected with PREX2a siRNA or non-specific siRNA (NC); and (B) in MG63 cells transfected with pcDNA3.1-PREX2a plasmid or blank pcDNA3.1 vector (NC). Control group: Untransfected cells. Data are presented as the mean \pm standard deviation. ${ }^{* *} \mathrm{P}<0.01$ vs. the control cells. PREX2a, phosphatidylinositol-3,4,5-trisphosphate-dependent Rac exchange factor $2 \mathrm{a}$; NC, negative control; siRNA, small interfering RNA; GAPDH, glyceraldehyde 3-phosphate dehydrogenase; MMP, matrix metalloproteinase.

PREX2a promotes the invasion and migration of OS cells. Since tumor cell invasion and migration have essential roles in cancer metastasis, the role of PREX2a was investigated in the regulation of OS cell invasion and migration, as determined by Transwell and wound healing assays, respectively. As shown in Fig. 4A and B, the invasive capacity of MG63 OS cells was significantly reduced following knockdown of PREX2a; however, PREX2a overexpression increased the invasion of MG63 cells. Similarly, cell migration was downregulated following knockdown of PREX2a expression, whereas it was increased following overexpression of PREX2a in MG63 cells (Fig. 4C and D). In addition, since MMP2 and MMP9 have important roles in mediating tumor cell invasion and migration, the expression levels of these proteins were detected in each group. Consistent with the results of the cell invasion and migration assays, knockdown of PREX2a inhibited the protein expression levels of MMP2 and MMP9, whereas overexpression of PREX2a enhanced their protein expression levels (Fig. 5A and B). These results indicate that PREX2a may have a promoting role in the regulation of OS cell invasion and migration, at least partly via modulation of MMP2 and MMP9 expression.

siRNA-mediated knockdown of PREX2a inhibits PI3K signaling activity in OS cells. PTEN and PI3K activity was further investigated in the MG63 OS cells following knockdown or overexpression of PREX2a. In MG63 cells overexpressing PREX2a, the phosphorylation levels of PTEN were increased, which was accompanied by upregulated phosphorylation of PI3K, thus indicating that PI3K signaling was activated (Fig. 6). Conversely, the phosphorylation levels of PTEN were reduced in MG63 cells transfected with PREX2a-specific siRNA, indicating that the activity of PTEN was upregulated, which was accompanied by downregulated phosphorylation of PI3K, thus indicating that PI3K signaling activity was reduced (Fig. 6). These results suggest that PTEN and PI3K signaling activity may be modulated by PREX2a in MG63 cells.

\section{Discussion}

The identification of novel oncogenes may aid in the development of therapeutic strategies against OS. The present study is

\section{Control PREX2a PREX2a siRNA}
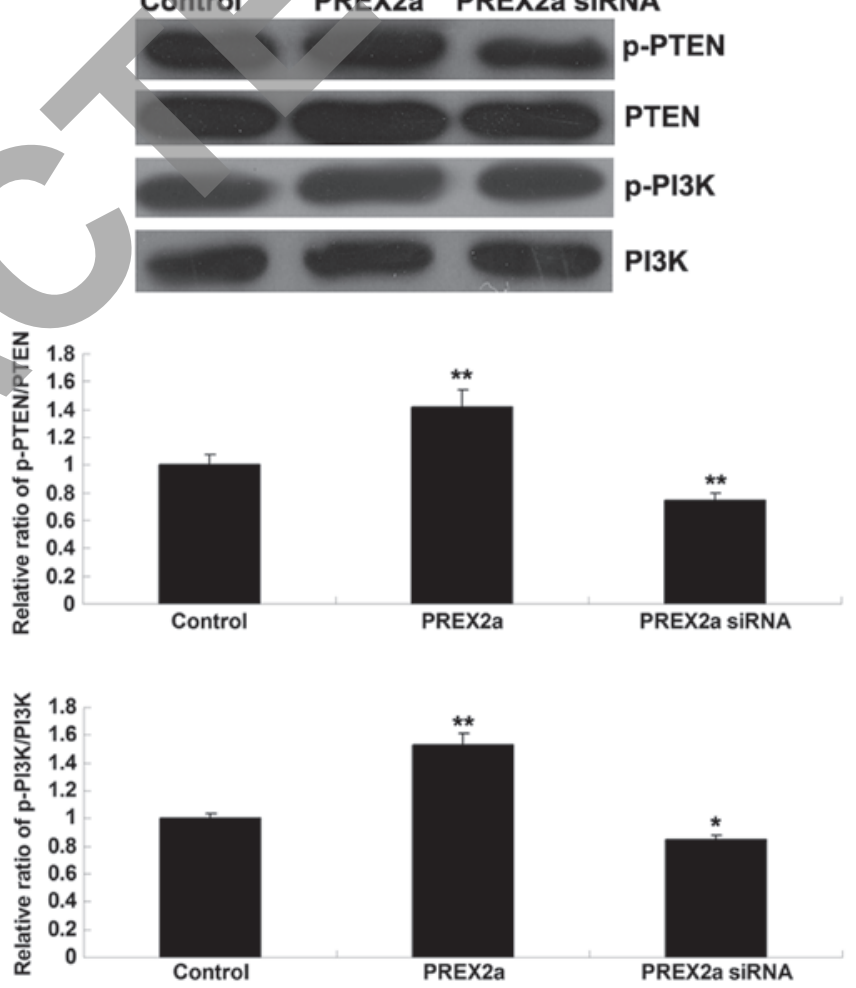

Figure 6 . Western blotting was performed to determine the protein expression levels of p-PTEN, PTEN, p-PI3K and PI3K in MG63 cells following knockdown or overexpression of PREX2a. Control group: Untransfected cells. Data are presented as the mean \pm standard deviation. ${ }^{*} \mathrm{P}<0.05$ and ${ }^{* *} \mathrm{P}<0.01$ vs. the control cells. PREX2a, phosphatidylinositol-3,4,5-trisphosphate-dependent Rac exchange factor 2a; siRNA, small interfering RNA; p-, phosphorylated; PTEN, phosphatase and tensin homolog deleted on chromosome ten; PI3K, phosphoinositide 3-kinase.

the first, to the best of our knowledge, to investigate the role of PREX2a in OS in vitro. The results demonstrated that PREX2a was significantly upregulated in OS cells, as compared with in normal human osteoblast cells. Further investigations revealed that PREX2a was able to promote OS cell proliferation, invasion and migration, potentially via upregulation of MMP2 and MMP9. In addition, the present study suggested that the oncogenic role of PREX2a in OS may be realized via direct 
inhibition of PTEN activity and subsequent activation of PI3K signaling.

PREX2a contains an N-terminal Dbl homology and pleckstrin homology domain, which confers GEF activity; pairs of PDZ and Dishevelled, Egl-10 and Pleckstrin domains; and a C-terminus with weak similarity to inositol 4-polyphosphate phosphatase (12). Through its GEF domains, PREX2a is able to directly bind to PTEN, and act as an inhibitor of PTEN activity $(18,19)$. Furthermore, the genetic sequence of PREX2a is similar to that of PREX1, which has been demonstrated to have an oncogenic role in human cancer. Qin et al (20) reported that upregulation of PREX1 enhanced prostate cancer metastasis; the expression of recombinant PREX1 in non-metastatic prostate cancer cells increased cell migration and invasion via Rac-dependent lamellipodia formation. In addition, using a mouse xenograft model, the expression of recombinant PREX1 was shown to induce lymph node metastasis of non-metastatic prostate cancer cells without an effect on primary tumor growth (20). Furthermore, Montero et al (21) identified a significant correlation between high PREX1 expression and poor patient outcome in breast cancer; knockdown of PREX1 expression suppressed breast cancer cell migration and invasion, and tumorigenic potential in vivo.

It has previously been reported that loss of PTEN function frequently occurs in human cancer; therefore, the binding partners of PTEN may also be involved in tumorigenesis via modulation of PTEN activity (10). Similar to PREX1, PREX2a has been demonstrated to act as an oncogene in human cancer via regulation of biological processes, including cell proliferation, cell cycle progression, migration and invasion $(14,15,22)$. Whole-genome sequencing identified PREX2 as a significantly mutated gene in melanoma (16). In addition, overexpression of PREX has been shown to be associated with poor patient outcome in breast cancer (23). PREX2a has also been identified as a direct target of microRNA (miR)-338-3p, and knockdown of PREX $2 \mathrm{a}$ inhibited cell proliferation and clonogenicity, and induced a $G_{1} / S$ arrest and apoptosis in gastric cancer cells, potentially via mediation of PTEN-PI3K signaling (14). Chen et al (15) also reported similar findings in neuroblastoma; knockdown of PREX2a inhibited neuroblastoma cell proliferation, migration and invasion via the PTEN-PI3K pathway. Furthermore, PREX2a was identified as a target gene of miR-338-3p, and overexpression of PREX2a reversed the inhibitory effects on the proliferation and invasion of neuroblastoma cells (15).

In the present study, PREX2a was shown to be significantly upregulated in OS cell lines, as compared with in a normal osteoblast cell line. In addition, silencing PREX2a expression suppressed OS cell proliferation, migration and invasion. MMP2 and MMP9 have been suggested to participate in the metastasis of OS (24), and the expression and activity of MMP2 and MMP9 are tightly associated with OS cell migration and invasion (25-27). The present study demonstrated that suppression of PREX2a notably inhibited the invasion and migration of OS cells, at least partly via suppressing the protein expression of MMP2 and MMP9.

In conclusion, the present study indicated that PREX2a had an oncogenic role in the regulation of proliferation, invasion and migration of OS cells via mediation of PI3K signaling.

\section{Acknowledgements}

The present study was supported by the Fundamental Research Funds for the Central Universities of Central South University (grant no. 2013zzts092).

\section{References}

1. Thompson LD: Osteosarcoma. Ear Nose Throat J 92: 288-290, 2013.

2. PosthumaDeBoer J, Witlox MA, Kaspers GJ and van Royen BJ: Molecular alterations as target for therapy in metastatic osteosarcoma: A review of literature. Clin Exp Metastasis 28: 493-503, 2011.

3. Yang J and Zhang W: New molecular insights into osteosarcoma targeted therapy. Curr Opin Oncol 25: 398-406, 2013.

4. Namløs HM, Meza-Zepeda LA, Barøy T, Østensen IH, Kresse SH, Kuijjer ML, Serra M, Bürger H, Cleton-Jansen AM and Myklebost O. Modulation of the osteosarcoma expression phenotype by microRNAs. PLOS One 7: e48086, 2012.

5. Braccini L, Ciraolo E, Martini M, Pirali T, Germena G, Rolfo K and Hirsch E: PI3K keeps the balance between metabolism and cancer. Adv Biol Regul 52: 389-405, 2012.

6. Wu P and Hu YZ: PI3K/Akt/mTOR pathway inhibitors in cancer: A perspective on clinical progress. Curr Med Chem 17: 4326-4341, 2010

7. Burris HA III: Overcoming acquired resistance to anticancer therapy: Focus on the PI3K/AKT/mTOR pathway. Cancer Chemother Pharmacol 71: 829-842, 2013.

8. Perry JA, Kiezun A, Tonzi P, Van Allen EM, Carter SL, Baca SC Cowley GS, Bhatt AS, Rheinbay E, Pedamallu CS, et al: Complementary genomic approaches highlight the PI3K/mTOR pathway as a common vulnerability in osteosarcoma. Proc Natl Acad Sci USA 111: E5564-E5573, 2014.

9. Yang L, Shu T, Liang Y, Gu W, Wang C, Song X, Fan C and Wang W: GDC-0152 attenuates the malignant progression of osteosarcoma promoted by ANGPTL2 via PI3K/AKT but not p38MAPK signaling pathway. Int J Oncol 46: 1651-1658, 2015.

10. Eng C: PTEN: One gene, many syndromes. Hum Mutat 22: 183-198, 2003.

11. Hodakoski C, Hopkins BD, Barrows D, Mense SM, Keniry M, Anderson KE, Kern PA, Hawkins PT, Stephens LR and Parsons R: Regulation of PTEN inhibition by the pleckstrin homology domain of P-REX2 during insulin signaling and glucose homeostasis. Proc Natl Acad Sci USA 111: 155-160, 2014.

12. Donald S, Hill K, Lecureuil C, Barnouin R, Krugmann S, John Coadwell W, Andrews SR, Walker SA, Hawkins PT, Stephens LR and Welch HC: P-Rex2, a new guanine-nucleotide exchange factor for Rac. FEBS Lett 572: 172-176, 2004.

13. Fine B, Hodakoski C, Koujak S, Su T, Saal LH, Maurer M, Hopkins B, Keniry M, Sulis ML, Mense S, et al: Activation of the PI3K pathway in cancer through inhibition of PTEN by exchange factor P-REX2a. Science 325: 1261-1265, 2009.

14. Guo B, Liu L, Yao J, Ma R, Chang D, Li Z, Song T and Huang C: miR-338-3p suppresses gastric cancer progression through a PTEN-AKT axis by targeting P-REX2a. Mol Cancer Res 12: 313-321, 2014.

15. Chen X, Pan M, Han L, Lu H, Hao X and Dong Q: miR-338-3p suppresses neuroblastoma proliferation, invasion and migration through targeting PREX2a. FEBS Lett 587: 3729-3737, 2013.

16. Berger MF, Hodis E, Heffernan TP, Deribe YL, Lawrence MS, Protopopov A, Ivanova E, Watson IR, Nickerson E, Ghosh P, et al: Melanoma genome sequencing reveals frequent PREX2 mutations. Nature 485: 502-506, 2012.

17. Livak KJ and Schmittgen TD: Analysis of relative gene expression data using real-time quantitative PCR and the 2(-Delta Delta C(T)) Method. Methods 25: 402-408, 2001.

18. Rosenfeldt H, Vázquez-Prado J and Gutkind JS: P-REX2, a novel PI-3-kinase sensitive Rac exchange factor. FEBS Lett 572: 167-171, 2004.

19. Leslie NR: P-REX2a driving tumorigenesis by PTEN inhibition. Sci Signal 2: pe68, 2009.

20. Qin J, Xie Y, Wang B, Hoshino M, Wolff DW, Zhao J, Scofield MA, Dowd FJ, Lin MF and Tu Y: Upregulation of PIP3-dependent Rac exchanger 1 (P-Rex1) promotes prostate cancer metastasis. Oncogene 28: 1853-1863, 2009. 
21. Montero JC, Seoane S, Ocaña A and Pandiella A: P-Rex1 participates in Neuregulin-ErbB signal transduction and its expression correlates with patient outcome in breast cancer. Oncogene 30: 1059-1071, 2011.

22. Donald S, Humby T, Fyfe I, Segonds-Pichon A, Walker SA, Andrews SR, Coadwell WJ, Emson P, Wilkinson LS and Welch HC: P-Rex 2 regulates Purkinje cell dendrite morphology and motor coordination. Proc Natl Acad Sci USA 105: 4483-4488, 2008.

23. Pandiella A and Montero JC: Molecular pathways: P-Rex in cancer. Clin Cancer Res 19: 4564-4569, 2013.

24. Wang JY, Wu PK, Chen PC, Yen CC, Hung GY, Chen CF, Hung SC, Tsai SF, Liu CL, Chen TH and Chen WM: Manipulation therapy prior to diagnosis induced primary osteosarcoma metastasis - from clinical to basic research. PLoS One 9: e96571, 2014.
25. Fromigué O, Hamidouche $\mathrm{Z}$ and Marie PJ: Blockade of the RhoA-JNK-c-Jun-MMP2 cascade by atorvastatin reduces osteosarcoma cell invasion. J Biol Chem 283: 30549-30556, 2008.

26. Chueh FS, Chen YY, Huang AC, Ho HC, Liao CL, Yang JS Kuo CL and Chung JG: Bufalin-inhibited migration and invasion in human osteosarcoma U-2 OS cells is carried out by suppression of the matrix metalloproteinase-2, ERK, and JNK signaling pathways. Environ Toxicol 29: 21-29, 2014.

27. Jin J, Cai L, Liu ZM and Zhou XS: miRNA-218 inhibits osteosarcoma cell migration and invasion by down-regulating of TIAM1, MMP2 and MMP9. Asian Pac J Cancer Prev 14: $3681-3684,2013$ 